\title{
Variabilidad genética en el pejerrata Caelorinchus fasciatus (Günther, 1878). (Pisces, Gadiformes, Macrouridae)
}

\author{
Genetic variability in the rat tail fish Caelorinchus fasciatus (Günther, 1878). \\ (Pisces, Gadiformes, Macrouridae) \\ Ciro Oyarzún ${ }^{1}$, Javier Monsalves ${ }^{2}$, Ricardo Galleguillos ${ }^{1}$ y Joel Sandoval \\ ${ }^{1}$ Sección Pesquerias, Departamento Oceanografia, Universidad de Concepción, Casilla 160-C, Concepción, Chile. \\ 2 Programa Doctorado en Ciencias Ambientales, Centro EULA-CHILE, Universidad de Concepción, Casilla 160-C, Chile.
}

coyarzun@udec.cl

\begin{abstract}
Resumen.- El ambiente marino de profundidad plantea interesantes situaciones para el estudio de procesos microevolutivos, permitiendo confrontar hipótesis que ven a esos ambientes como muy estables o bien sujetos a perturbaciones periódicas a las que las especies responderán según su potencial genético y limitantes filogenéticas. En ese contexto se estudió la variabilidad genética en un peje-rata de la costa de Chile: Caelorinchus fasciatus. Mediante electroforesis en gel de almidón se evidenciaron los productos enzimáticos de 28 loci presuntivos, de los cuales, sólo tres resultaron polimórficos (PGI-1, PGI-2 y $P G M-1)$. La baja variabilidad genética encontrada $(\mathrm{H}=0,026)$ es propia de especies generalistas de hábitat. Comparado con Macrourus holotrachys, la única especie chilena de la familia de la cual se tiene información genética, 12 loci muestran una expresión semejante en ambas especies, incluyendo 5 loci de proteínas totales; la Identidad Génica entre ambas especies alcanzó valores de $\mathrm{I}=0,409$ y la Distancia fue $\mathrm{D}=0,894$. Además, los resultados del presente trabajo, apoyan la Hipótesis de Gauldie, de encontrar baja variabilidad en los sistemas enzimáticos PGI y PGM o sólo en uno de ellos, pero nunca alta en ambos a la vez.

Palabras clave: peces de profundidad, heterocigosidad, Pacífico Suroriental, Chile.
\end{abstract}

\begin{abstract}
Studies on microevolutionary processes in deep-sea environments, allow testing the contrasting hypotheses about the environmental stability. Some of these consider those environments as stable or suffering periodical disturbances. In those cases the species will answer according to its genetic potential and phylogenetic constrains. In this context, the genetic variability in the rat tail fish Caelorinchus fasciatus was studied. Using starch electrophoresis the enzymatic products of 28 presumptive loci were screened; only three of them were polymorphic PGI-1, PGI-2 and PGM1. The low genetic variability found $(\mathrm{H}=0,026)$ is seen as typical of habitat generalist species. Compared with Macrourus holotrachys, the single Chilean species that has genetic records, 12 loci show similar electrophoretic expression in both species, including 5 loci of total proteins. The Genetic Identity between both species was $\mathrm{I}=0,409$ and the Genetic Distance was $\mathrm{D}=0,894$. Moreover, our results support the Gauldie's hypothesis that it is possible to find low variability in the enzymatic systems $P G I$ and $P G M$, or in one of them but never high variability in both of them simultaneously.

Keywords: deep-sea fishes, heterozigosity, Southeastern Pacific, Chile.
\end{abstract}

\section{Introducción}

En el mar profundo habitan numerosas especies que forman comunidades donde ha ocurrido la evolución de importantes taxa superiores (Hessler \& Wilson 1983). Sin embargo, el conocimiento de los procesos de especiación en dicho ambiente, presenta serios problemas epistemológicos y técnicos que dificultan su avance, esto es especialmente grave en el grupo de los Macroúridos o de los Gadiformes en general, cuya sistemática se encuentra en permanente revisión (Cohen 1989).

Dentro del grupo de los Gadiformes, Macrouridae constituye la familia más numerosa, abarcando unas 300 especies de hábitats principalmente bentopelágicos, siendo especies exclusivas de aguas profundas (Oyarzún et al. 1993). Asimismo, la existencia de dicho grupo en Chile, con una marcada presencia en biomasa y recurrencia a lo largo de la costa chilena (Oyarzún et al. $1989^{1}$ ), ha hecho necesaria continuas revisiones de la familia para las aguas del mar de Chile. Así en 1971, Pequeño reconoce la existencia de 11 especies en 5 géneros, posteriormente en su listado de todos los peces de Chile, la lista crece a 28 especies en 11 géneros (Pequeño 1989), en tanto que en la Addenda a dicho

\footnotetext{
${ }^{1}$ Oyarzún, C., I. Kong \& P. Aroca. 1989. La asociación de peces del talud continental de Chile, entre Arica e isla Mocha. IX Jornadas de Ciencias del Mar, Libro de Resumenes, C-42, p. 105 .
} 
trabajo, se agregan otras 25 especies, lo que daría un total de 53 especies (Pequeño 1997).

En este contexto, establecer la variabilidad genética de uno de los grupos más representativos del mar profundo, como Macrouridae, constituye el primer paso para ayudar a esclarecer los procesos evolutivos que conformaron la diversidad de fauna de los ambientes marinos de profundidad. Dado que el control genético simple de variantes proteínicas permite una aproximación directa a la variación genética dentro y entre especies (Ferguson \& Fleming 1983), las estimaciones de la diferenciación genética a lo largo del rango de distribución de las especies marinas ha sido uno de los campos más activos de la investigación genética en este ambiente (Koehn 1984; Ward et al. 1994).

Dado lo anterior, en este trabajo se presentan los resultados del estudio de la variabilidad genética en un pejerrata de la costa de Chile: Caelorinchus fasciatus (Günther, 1878), a través del análisis electroforético de proteínas.

Para fines comparativos, se utilizó la especie Macrourus holotrachys Günther, 1878, por ser el único representante de la familia que habita las costas de Chile y para la cual se cuenta con información de su variabilidad genética (Oyarzún et al. 1993) y cuya distribución se restringe a profundidades mayores a los $700 \mathrm{~m}$.

\section{Materiales y Métodos}

Se analizaron 42 ejemplares de $C$. fasciatus, provenientes del sector externo de la bahía de Concepción ( $36^{\circ} 35^{\prime} \mathrm{S}$ y $73^{\circ} 10^{\prime} \mathrm{W}$ ) capturados mediante red de arrastre a una profundidad de $300 \mathrm{~m}$ aproximadamente, como fauna concurrente de la pesca de langostino (Cervimunida johni Porter, 1903), en julio de 1992.

Como grupo comparativo externo y para la homologización de sistemas se utilizaron 10 ejemplares de $M$. holotrachys, provenientes de la pesca artesanal con espinel de profundidad entre los 800 y los $1200 \mathrm{~m}$ obtenidos en la misma fecha anterior.

De cada uno de los ejemplares congelados se obtuvieron muestras de músculo e hígado, las que fueron individualizadas y rotuladas en bolsas plásticas, para luego ser almacenadas junto a los ejemplares a $20^{\circ} \mathrm{C}$ hasta su análisis electroforético.

Las condiciones de corridas electroforéticas, tiempos, tampones y tinciones específicas, se explican en detalle en Oyarzún et al. (1993). Para la denominación de los alelos se siguió a Shaklee et al. (1990). Se tomó como especie patrón a M. holotrachys designando al alelo más frecuente como 100. Los demás alelos fueron numerados según su movilidad relativa a dicho alelo patrón. En el caso de enzimas con más de un locus, se enumeraron consecutivamente a partir del locus con la mayor migración anódica.

La electroforesis enzimática resolvió un total de 38 loci presuntivos (28 loci en músculo y 10 loci en el hígado, ver Tabla 1). Además, permitió establecer el número de individuos de los distintos genotipos obtenidos en las muestras de cada especie. Esta información se ingresó al programa computacional BIOSYS versión 1.7 (Swofford \& Selander 1989), mediante el cual se calcularon las frecuencias alélicas con su ajuste al equilibrio Hardy-Weinberg, la heterocigosidad media $(\mathrm{H})$ por locus y por individuo para cada especie (Tabla 2). Finalmente, para $M$. holotrachys se utilizó la información disponible previamente de Oyarzún et al. 1993.

\section{Resultados}

La electroforesis enzimática reveló un total de 28 loci presuntivos en músculo (correspondientes a 14 sistemas enzimáticos y a proteínas totales), con sólo 3 loci polimórficos: $P G I-1, P G I-2$ y $P G M-1$; en tanto que en el hígado se resolvieron 10 loci presuntivos (provenientes de 6 sistemas enzimáticos), ninguno de ellos polimórfico. Algunos sistemas presentaron actividad en ambos tejidos, en tanto que la $I D H$ fue exclusiva del tejido hepático. Los loci resueltos, los tampones y tejidos usados se señalan en la Tabla 1.

Las frecuencias alélicas de los sistemas polimórficos se describen en la Tabla 2 , donde se señala también que los valores de $\mathrm{Chi}^{2}$ para los loci PGI-1, PGI-2 y PGM-1 no mostraron desviaciones significativas del equilibrio de Hardy-Weinberg.

Respecto de los valores de variabilidad genética, medida como la heterocigosidad observada media (Ho) por individuo por locus, C. fasciatus alcanza un valor de $\mathrm{Ho}=0,026$. Mientras que al analizar sólo los loci polimórficos, los valores de heterocigosidad alcanzaron para $P G I-1$ una $\mathrm{Ho}=0,33$ y para $P G I-2$ una $\mathrm{Ho}=0,111$, lo que entrega un promedio para el sistema $P G I$ de $\mathrm{Ho}=0,222$, por su parte $P G M-1$ alcanza una $\mathrm{Ho}=0,277$ (Tabla 2).

De la comparación entre $C$. fasciatus y $M$. holotrachys (Tabla 3), se desprende que presentan actividad comparable en 11 sistemas y 17 loci enzimáticos. Debe agregarse a lo anterior 6 loci de Proteínas Totales. De los sistemas enzimáticos, se presentaron con expresión idéntica los loci monomórficos EM-1, EST-2, $A P-2, C A-1$ y $C A-2$. De los loci polimórficos, en $P G M-1$ ambas especies comparten los alelos 90 y 95. En cuanto a Proteínas Totales, se presentan en forma idéntica cinco de seis sistemas resueltos. 
Tabla 1

Código internacional, número de loci obtenidos, tampón utilizado y polimorfismo que presenta cada una de las enzimas en la especie $C$. fasciatus. *= locus polimórfico.

International code, amount of loci screened, buffer used and level of polymorphism at each enzyme in $C$. fasciatus. *= polymorphic locus.

\begin{tabular}{|c|c|c|c|c|c|c|}
\hline $\mathrm{CI}$ & E N Z I M A S & ABREV & LOCUS & $\mathrm{N}^{\circ} \mathrm{LOCI}$ & BUFFER & TEJIDO \\
\hline 1.1.1.8 & $\alpha$-glicerofosfato deshidrogenasa & $\alpha \mathrm{GPDH}$ & $\alpha G P D H-1$ & 1 & TC 8,0 & M \\
\hline 1.1.1.8 & Glicerol-3-fosfato & G3PDH & $G 3 P D H-1$ & 1 & $\mathrm{LIOH}$ & M \\
\hline \multirow[t]{3}{*}{1.1 .1 .27} & Lactato deshidrogenasa & LDH & $L D H-1,2$ & 3 & $\mathrm{LIOH}$ & $\mathrm{H}$ \\
\hline & & & $L D H-C$ & & & \\
\hline & & & $L D H-1,2$ & 2 & $\mathrm{LIOH}$ & M \\
\hline 1.1.1.39 & Malato deshidrogenasa & $\mathrm{MDH}$ & $M D H-1$ & 1 & TC 6,9 & M \\
\hline 1.1.1.40 & Enzima málica & EM & $E M-1$ & 1 & BORATO & M \\
\hline 1.1.1.42 & Isocitrato deshidrogenasa & IDH (ICD) & $I D H-1$ & 1 & TC 5,1-6,0 & $\mathrm{H}$ \\
\hline \multirow[t]{2}{*}{ 1.15.1.1. } & Tetrazolium oxidasa & $\mathrm{TO}$ & $T O-1$ & 2 & TC 5,1-6,0 & M \\
\hline & & & TO-2 & & & \\
\hline 2.7.3.2 & Creatina kinasa & $\mathrm{CK}$ & $C K-1$ & 1 & TC 8,0 & M \\
\hline 2.7.5.1 & Fosfogluco mutasa & PGM & $P G M-1$ & 1 & TC 8,0 & $M^{*}$ \\
\hline \multirow[t]{2}{*}{ 3.1.1.1 } & Esterasas & EST & $E S T-1,2$ & 2 & $\mathrm{LIOH}$ & $\mathrm{H}$ \\
\hline & & & $E S T-1,2,3$ & 3 & $\mathrm{LIOH}$ & M \\
\hline 3.4 .11 .1 & Leucin aminopeptidasa & LAP & $L A P-1$ & 1 & POULIK & $\mathrm{M}, \mathrm{H}$ \\
\hline \multirow[t]{2}{*}{3.4 .13} & Amino peptidasa & $\mathrm{AP}$ & $A P-1,2,3$ & 3 & $\mathrm{LIOH}$ & M \\
\hline & & & $A P-1,2$ & 2 & $\mathrm{LIOH}$ & $\mathrm{H}$ \\
\hline \multirow[t]{2}{*}{ 4.2.1.1 } & Anhidrasa carbonica & $\mathrm{CA}$ & $C A-1,2$ & 2 & TC 8,0 & M \\
\hline & & & $C A-1$ & 1 & TC80 & $\mathrm{H}$ \\
\hline 4.4.1.5 & Glioxalasa & GLIO & GLIO-1 & 1 & POULIK & M \\
\hline \multirow[t]{2}{*}{ 5.3.1.9. } & Fosfoglucoisomerasa & PGI & $P G I-1$ & 2 & TC 5,1-6,0 & $M^{*}$ \\
\hline & & & $P G I-2$ & & & \\
\hline --------- & Proteinas totales & PT & $P T-1-6$ & 6 & $\mathrm{LIOH}$ & M \\
\hline
\end{tabular}

T.C.8.0 : $\quad$ Tris Cítrico $\mathrm{pH} \mathrm{8,0}$

LIOH : Hidróxido de Litio $\mathrm{pH} 8,31-8,34$

T.C. 6,9 Tris Cítrico $\mathrm{pH} \mathrm{6,9}$

Borato : Ac. Bórico $\mathrm{pH} \mathrm{8,6}$

T.C. 5.1-6.0 Tris Cítrico Discontinuo $\mathrm{pH} 5,1-6,0$

Poulik : Poulik Discontinuo pH 8,7-8,2 
Tabla 2

Frecuencia alélica para cada locus polimórfico, Chi cuadrado para ajuste a Hardy-Weinberg y el número de genotipos encontrados en la muestra de $C$. fasciatus.

Allelic frequency, genotypic abundance, Chi-square test for Hardy-Weinberg equilibrium, for polymorphic loci in C. fasciatus

\begin{tabular}{|c|c|c|c|c|c|c|}
\hline LOCUS & GENOTIPOS & $\mathrm{N}^{\circ} \mathrm{OBS}(\mathrm{ESP})$ & ALELOS & FREC & Ho & $\mathrm{H}-\mathrm{W}$ \\
\hline \multirow[t]{3}{*}{$P G I-1$} & $120 / 120$ & $11(10,8)$ & 120 & 0,778 & 0,333 & $\mathrm{p}=0,779$ \\
\hline & $120 / 130$ & $6(6,4)$ & 130 & 0,222 & & \\
\hline & $130 / 130$ & $1(0,8)$ & & & & \\
\hline \multirow[t]{3}{*}{$P G I-2$} & $200 / 200$ & $16(16,03)$ & 200 & 0,944 & 0,111 & $\mathrm{p}=0,862$ \\
\hline & $200 / 210$ & $2(1,94)$ & 210 & 0,056 & & \\
\hline & $210 / 210$ & $0(0,03)$ & & & & \\
\hline \multirow[t]{6}{*}{$P G M-1$} & $85 / 85$ & $13(13,29)$ & 85 & 0,861 & 0,277 & $\mathrm{p}=0,947$ \\
\hline & $85 / 90$ & $4(3,54)$ & 90 & 0,111 & & \\
\hline & $85 / 95$ & $1(0,89)$ & 95 & 0,028 & & \\
\hline & $90 / 90$ & $0(0,17)$ & & & & \\
\hline & $90 / 90$ & $0(0,11)$ & & & & \\
\hline & $90 / 90$ & $0(0,00)$ & & & & \\
\hline
\end{tabular}

Heterocigosidad Media $\quad 0,240$

Cabe destacar la migración catódica que presentó el sistema LDH-C (sensu Shaklee \& Whitt, 1981) en el hígado de todos los ejemplares analizados para ambas especies.

Finalmente se calcularon los valores de Identidad $(\mathrm{I}=0.409)$ y Distancia $(\mathrm{D}=0.894)$ que corresponden con el nivel de separación taxonómica (géneros distintos) de las especies estudiadas.

\section{Discusión y Conclusiones}

De acuerdo a lo propuesto por Siebenaller (1978), las especies que habitan el mar profundo deberían presentar una menor variabilidad genética por habitar ambientes presuntamente más homogéneos y uniformes, recordando que hasta hace un par de décadas, el mar por debajo de los $200 \mathrm{~m}$ era considerado como una región extremadamente homogénea y estable, el paradigma mismo de la estabilidad ambiental (Gooch \& Schopf 1973).

Una explicación alternativa es la presentada por Smith \& Fujio (1982) de que las especies especialistas alcanzarían altos niveles de variabilidad genética y que especies generalistas, por contraparte, tendrían bajos niveles de variabilidad genética (Smith \& Fujio, 1982). Por otro lado se ha postulado que la baja variabilidad genética característica del Orden, se debería más bien a restricciones filogenéticas que a determinantes ambientales (Oyarzún et al. 1997).
En la especie analizada (C. fasciatus) se confirma la baja variabilidad del grupo, ya que de 28 loci evidenciados, sólo tres de ellos muestran polimorfismo, alcanzando un valor bajo de heterocigocidad según lo señalado en literatura (Smith \& Fujio 1982). Incluso dos de aquellos loci pertenecen al sistema PGI. Consistentemente, junto al sistema $P G M$, son los mismos sistemas que han mostrado variación en $M$. holotrachys y Antimora rostrata (Günther, 1878) independiente de los muy diferentes números muestreales analizados en cada caso (Oyarzún et al. 1993, 1995).

Otro aspecto particular es la expresión del sistema $L D H-C$, que a diferencia de los otros loci, tiene una migración catódica y con su máxima expresión en el tejido hepático. Nuestros resultados confirman esa expresión, agregando nueva evidencia a favor de la hipótesis de que corresponde a un carácter conservativo, propio del grupo Gadiformes (sinapomorfía del grupo), pero derivado respecto del resto de los teleósteos (Patterson \& Rosen, 1989).

Los resultados de la presente investigación confirman la característica del grupo, al presentar bajos niveles de variabilidad genética similares a los reportados para $C$. armatus (Hector, 1875) tanto en el Pacífico Norte como en el Atlántico Norte (Wilson \& Waples 1984) y para C. yaquinae (Iwamoto \& Stein, 1974) del Pacífico Norte (Wilson \& Waples 1983). A esto se agrega, la información de reportes previos en 
especies chilenas de Gadiformes (Oyarzún et al. 1993, Oyarzún et al. 1997).

Resulta interesante destacar, que a pesar de estar comparando especies pertenecientes a dos géneros (Caelorinchus y Macrourus), existe una proporción importante de sistemas enzimáticos que presentan idéntica movilidad, aun cuando se presenten monomórficos (ver Tabla 3). Lo anterior, explicaría los valores de Identidad y Distancia encontrados, que alcanzan los niveles habituales de especies o géneros estrechamente relacionados (Ayala 1983).

Gauldie (1984) ha planteado una hipótesis según la cual existiría una relación entre la variabilidad genética de los sistemas $P G M$ y $P G I$, donde pueden encontrarse altos valores de variabilidad en uno de los sistemas, o bien ser baja en ambos, pero nunca alta en los dos sistemas simultáneamente. Los resultados aquí presentados refuerzan la evidencia en el sentido de la relación existente entre los niveles de variabilidad genética de estos loci, abriendo la discusión acerca del papel que juega la adaptación y los mecanismos selectivos, en el patrón descrito por Gauldie, ya que dicha relación aparece en especies generalistas tanto como en especialistas (sensu Smith \& Fujio 1982), asimismo aparece descrita para diversos ambientes, incluido el mar profundo. Los resultados presentados apoyan la idea de que cada gen no está evolucionando aisladamente, sino que por el contrario, es parte de un conjunto mayor que la selección pudiera haber ido moldeando en el tiempo, de acuerdo a los potenciales de variación propios de cada grupo taxonómico y a los desafíos ambientales que la especie debe enfrentar.

Tabla 3

\section{Comparación de las frecuencias alélicas obtenidas en músculo (M) e hígado (H) de M. holotrachys y C. fasciatus.}

Comparison of allelic frequencies found in muscle of $M$. holotrachys y $C$. fasciatus.

\begin{tabular}{|c|c|c|c|c|c|c|c|c|c|}
\hline LOCUS & ALELO & $\begin{array}{l}\text { M. holotrachys } \\
(\mathrm{n}=70)\end{array}$ & $\begin{array}{l}\text { C. fasciatus } \\
(\mathrm{n}=42)\end{array}$ & Tejido & LOCUS & ALELO & $\begin{array}{l}\text { M. holotrachys } \\
\quad(\mathrm{n}=70)\end{array}$ & $\begin{array}{l}\text { C. fasciatus } \\
(\mathrm{n}=42)\end{array}$ & Tejido \\
\hline \multirow[t]{2}{*}{ G3PDH-1 } & 100 & 1,0 & 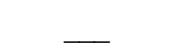 & M & & 95 & 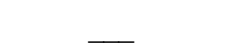 & 1,0 & \\
\hline & 70 & 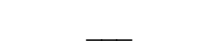 & 1,0 & & $A P-1$ & 120 & 1,0 & 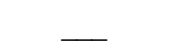 & $\mathrm{H}$ \\
\hline \multirow[t]{2}{*}{$L D H-1$} & 105 & 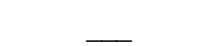 & 1,0 & M & & 100 & 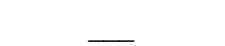 & 1,0 & \\
\hline & 100 & 1,0 & 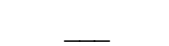 & & $A P-2$ & 100 & 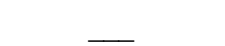 & 1,0 & $\mathrm{H}$ \\
\hline \multirow[t]{2}{*}{$L D H-2$} & 100 & 1,0 & _ & M & C.A.-1 & 100 & 1,0 & 1,0 & M \\
\hline & 90 & 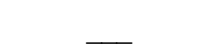 & $\overline{1,0}$ & & C.A.-2 & 100 & 1,0 & 1,0 & M \\
\hline \multirow[t]{2}{*}{$L D H-1$} & 105 & 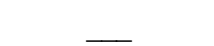 & 1,0 & $\mathrm{H}$ & C.A. -1 & 105 & 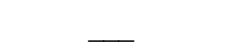 & 1,0 & $\mathrm{H}$ \\
\hline & 100 & 1,0 & - & & & 100 & 1,0 & 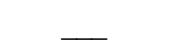 & \\
\hline \multirow[t]{2}{*}{$L D H-C$} & 105 & 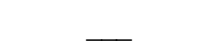 & 1,0 & $\mathrm{H}$ & C.A. -2 & 100 & 1,0 & 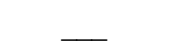 & $\mathrm{H}$ \\
\hline & 100 & 1,0 & 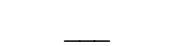 & & GLIO-1 & 100 & 1,0 & 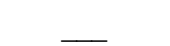 & M \\
\hline \multirow[t]{2}{*}{$M D H-1$} & 100 & 1,0 & 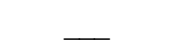 & M & & 95 & 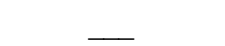 & 1,0 & \\
\hline & 90 & 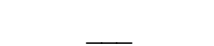 & $\overline{1,0}$ & & PGI-1 & 130 & - & 0,222 & M \\
\hline$M D H-1$ & 100 & 1,0 & 1,0 & $\mathrm{H}$ & & 120 & 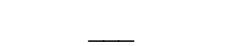 & 0,778 & \\
\hline E.M.-1 & 100 & 1,0 & 1,0 & M & & 110 & 0,22 & 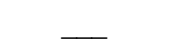 & \\
\hline \multirow[t]{2}{*}{ C.K.-1 } & 105 & 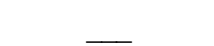 & 1,0 & M & & 100 & 0,53 & 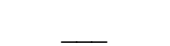 & \\
\hline & 100 & 1,0 & 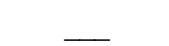 & & & 90 & 0,24 & $\ldots$ & \\
\hline \multirow[t]{6}{*}{$P G M-1$} & 110 & 0,15 & 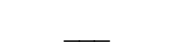 & M & $P G I-2$ & 210 & 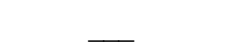 & 0,056 & M \\
\hline & 105 & 0,16 & $\bar{\tau}$ & & & 200 & $\overline{-}$ & 0,944 & \\
\hline & 100 & 0,43 & - & & & 110 & 0,03 & 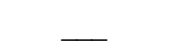 & \\
\hline & 95 & 0,12 & 0,028 & & & 100 & 0,97 & 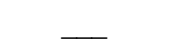 & \\
\hline & 90 & 0,13 & 0,111 & & P. T. -1 & 105 & 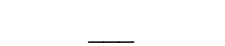 & 1,0 & M \\
\hline & 85 & 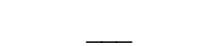 & 0,861 & & & 100 & $\overline{1,0}$ & 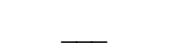 & \\
\hline \multirow[t]{2}{*}{ EST-1 } & 105 & 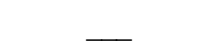 & 1,0 & $\mathrm{M}, \mathrm{H}$ & P.T.-2 & 100 & 1,0 & 1,0 & M \\
\hline & 100 & 1,0 & 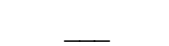 & & P.T.-3 & 100 & 1,0 & 1,0 & $\mathrm{M}$ \\
\hline EST-2 & 100 & 1,0 & 1,0 & $\mathrm{M}, \mathrm{H}$ & P.T.-4 & 100 & 1,0 & 1,0 & M \\
\hline$A P-1$ & 100 & 1,0 & 1,0 & M & P.T. -5 & 100 & 1,0 & 1,0 & M \\
\hline$A P-2$ & 100 & 1,0 & 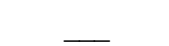 & $\mathrm{M}$ & P.T.-6 & 100 & 1,0 & 1,0 & M \\
\hline
\end{tabular}




\section{Agradecimientos}

Esta investigación contó con el financiamiento de los Proyectos FONDECYT 820-91 y 1950057. Se agradece a dos revisores anónimos que con sus sugerencias permitieron mejorar el trabajo.

\section{Literatura Citada}

Ayala FJ. 1983. Enzymes as taxonomic characters. En: Oxford, GS \& D Rollinson (eds), Protein Polymorphism: Adaptive and Taxonomic significance. Systematic Association, Special Volume 24: 3-26. Academic Press, London.

Cohen DM (ed). 1989. Papers on the systematics of Gadiform fishes. Natural History Museum of Los Angeles County, Sciences Series 32: 1-262.

Ferguson A \& CC Fleming. 1983. Evolutionary and taxonomic significance of protein variation in the Brown trout (Salmo trutta L.) and other Salmonid fishes. En: Oxford, GS \& D Rollinson (eds), Protein Polymorphism: Adaptive and Taxonomic significance. Systematic Association, Special Volume 24: 85-99. Academic Press, London.

Gauldie RW 1984. A reciprocal relationship between heterozygosities of the phosphoglucomutase and glucose phosphate isomerase loci. Genetica 63: 93-103.

Gooch JL \& TJM Schopf. 1973. Genetic variability in the deep-sea: relation to environmental variability. Evolution 26(4): 545-552.

Hessler RR \& GDF Wilson. 1983. The origin and biogeography of Malacostracan crustacean in the deep sea. En: Sims RW, JH Price \& PES Whalley (eds), Evolution, Time and Space: The emergence of Biosphere. Systematic Association, Special Volume 23: 227-254. Academic Press, London.

Koehn RK. 1984. The application of genetics to problems in the marine environment: future areas of research. Nerc, Swindon, U.K. 44 pp.

Oyarzún C, R Galleguillos \& J Monsalves. 1993. Variabilidad genética en el granadero de profundidad Macrourus holotrachys Günther, 1878, capturado en la zona pesquera de Talcahuano (Pisces, Gadiformes, Macrouridae). Revista de Biología Marina, Valparaíso 28(2): 331-340.

Oyarzún C, J Monsalves \& $\mathbf{R}$ Galleguillos. 1995. Variabilidad genética en un pez de profundidad Antimora rostrata (Günther 1878), capturado en la zona pesquera de Talcahuano (Pisces, Gadiformes, Moridae). Estudios Oceanológicos 14: 1-4.
Oyarzún C, R Galleguillos \& J Monsalves. 1997. Variabilidad genética en peces demersales: distribución en profundidad versus restricciones filogenéticas. Scientia Marina 61: 291-296.

Patterson C \& DE Rosen. 1989. The Paracanthopterygii revisited: order and disorder. En: DM Cohen (ed). Papers on the systematics of Gadiform fishes. Natural History Museum of Los Angeles County, Sciences Series 32:5-36.

Pequeño G. 1971. Sinopsis de Macrouriformes de Chile. Boletín del Museo Nacional de Historia Natural, Chile 32: 269-298.

Pequeño G. 1989. Peces de Chile. Lista sistemática revisada y comentada. Revista de Biología Marina, Valparaíso 24: 1132.

Pequeño G. 1997. Peces de Chile. Lista sistemática revisada y comentada: Addendum. Revista de Biología Marina y Oceanografía 32: 77-94.

Shaklee JB, FW Allendorf, DC Morizot \& GS Whitt. 1990. Gene nomenclature for Protein-coding loci in fish. Transactions of the American Fisheries Society 119: 2-15.

Shaklee JB \& GS Whitt. 1981. Lactate Dehydrogenase isozymes of Gadiform fishes: divergent patterns of gene expression indicate a heterogeneus taxon. Copeia 1981(3): 563-578.

Siebenaller JF. 1978. Variablity in Deep-Sea Fishes of the genus Sebastolobus (Scorpaenidae). En: Battaglia B \& JA Beardmore (eds), Marine Organisms, Genetics, Ecology, and Evolution. Vol 2: 123-140. Plenum Press, New York and London.

Smith PJ \& Y Fujio. 1982. Genetic variation in marine teleosts: High variability in habitat specialists and low variability in habitat generalists. Marine Biology 69: 7-20.

Swofford DL \& RB Selander. 1989. BIOSYS-1. A computer program for the analysis of allelic variation in population genetics and biochemical systematics. Illinois. Natural History Survey $43 \mathrm{pp}$.

Wilson GDF \& RR Hessler. 1987. Speciation in the deepsea. Annual Review of Ecology and Systematics 18: 185207.

Wilson RR \& RS Waples. 1983. Distribution, morphology, and biochemical genetics of Coryphaenoides armatus and C. yaquinae (Pisces: Macrouridae) in the Central and Eastern North Pacific. Deep-Sea Research 30:1227-1245.

Wilson RR \& RS Waples. 1984. Electrophoretic and biometric variability in the abyssal grenadier Coryphaenoides armatus of the western North Atlantic, eastern North Pacific Oceans. Marine Biology 80: 227237.

Ward RD, M Woodwark \& DOF Skibinski. 1994. A comparison of genetic diversity levels in marine, freshwater, and anadromous fishes. Journal of Fish Biology 44: 213-232. 